\title{
Individual Differences in Face Identification: A Causal Relationship with Anxiety?
}

\section{Ahmed M Megreya*}

Department of Psychological Sciences, College of Education, Qatar University, Qatar

Face identification has become one of the most popular topics in psychology, encompassing the cognitive, forensic, neuroscience, developmental, and social divisions. Most of this research, however, treats face identification as though all observers are equivalent, by studying the "average" human observer [1], ignoring the substantial individual differences that exist in the ability to process faces $[2,3]$. For example, using a simple face-matching task, in which observers have to decide if pairs of unknown faces depict the same person or two different people, individual performance ranges along a broad continuum from close-to-chance to perfect [4-8]. In addition, studies of recognition memory for unfamiliar faces reveal a similar distribution of ability, with individual $d$ ' scores ranging from 0.5 to 6.8 for old/new decisions to previously seen faces [9]. Understanding these individual differences is crucial for improving face identification and for enhancing security. Importantly, however, rather little is still known about their underlying causes.

Previous studies on unfamiliar face identification have focused on the properties of target stimuli to explore general differences, between groups or conditions, in performance. This is based on the observation that every encounter with a person provides a different pattern for visual analysis, due to, for example, changes in lighting, viewpoint, facial expression, and so forth. With limited familiarity of a face, it becomes difficult to dissociate visual information created by these contextual variables from the underlying structural information of a face. Many of these external factors are now well understood [10]. It seems unlikely, however, that any of these factors can account for individual differences in face identification, as viewing conditions and stimuli are typically held constant across participants. Understanding these individual differences therefore requires an alternative approach that focuses on internal factors, within observers, that may affect face processing.

Some of these factors have already been examined, although a complete picture remains elusive. Individual face recognition ability appears to be related to the individuals' race [11], gender [12,13] and age [5]. In addition to these factors, there is also evidence that a proficiency in face perception may reflect a general advantage in visual processing. This is borne out of the observation that people who are good at face recognition also appear to be good at object and scene perception [9]. For example, face-matching performance in a lineup task can be predicted by visual-short term memory, perceptual speed, and objectmatching ability [2]. Therefore a general proficiency in visual processing appears to underlie a good ability in face identification.

Intriguingly, several studies have explored whether face identification is also driven by factors that appear "non-visual" in nature. For example, some recent research reported that individual differences in face identification link to individual differences in a variety of personality traits, although those studies have yielded somewhat inconsistent results. Importantly however, research consistently showed that observers who exhibit high levels of neuroticism are less accurate at face processing than individuals with emotional stability $[3,14]$. Among the facets of neuroticism, anxiety revealed the most reliable associations with face identification [3]. For example, anxiety was found to degrade face memory [15] and face perception [16]. In addition, using eyewitness identification paradigm $[17,18]$, anxiety was found to impair eyewitness performances [19]. Therefore, psychological treatments of anxiety were found to improve face identification [16].

In a well-conducted study [20], the associations between face recognition and social anxiety were much stronger than those between face processing and general anxiety. Indeed, there might be a strong link between face processing and the dynamics of social communications. Specifically, individuals with high social anxiety might not have good eye contact skills, although the eyes were found to provide the most important information for face individuation [21]. However, the causal relationship between anxiety and face identification remains unclear and needs more attention by future studies.

\section{References}

1. Calder AJ, Rhodes G, Johnson MH, Haxby J (2011) The Oxford Handbook of Face Perception. Oxford: Oxford University Press.

2. Megreya AM, Burton AM (2006) Unfamiliar faces are not faces: Evidence from a matching task. Memory \& Cognition 4: 865-876.

3. Megreya AM, White D, Burton AM (2011) The other race effect does not rely on memory: evidence from a matching task. Quarterly Journal of Experimental Psychology 64: 1473-1483.

4. Megreya AM, Bindemann M, Havard C (2011) Sex differences in unfamiliar face identification: Evidence from matching tasks. Acta Psychologica 137: 83-89.

5. Megreya AM, Bindemann M (2015) Developmental improvement and agerelated decline in unfamiliar face matching. Perception 44: 2-44.

6. Li J, Tian M, Fang H, Xu M, Li H, et al. (2010) Extroversion predicts individual differences in face recognition. Communication \& Integrative Biology 3: 295298.

7. Megreya AM, Bindemann M (2013) Individual differences in personality and face identification. Journal of Cognitive Psychology 25: 30-37.

8. Mueller JH, Bailis KL, Goldstein AG (1979) Depth of processing and anxiety in facial recognition. British Journal of Psychology 70: 511-515.

9. Attwood AS, Penton-Voak IS, Burton AM, Munafò MR (2013) Acute Anxiety Impairs Accuracy in Identifying Photographed Faces. Psychological Science 24: 1591-1594.

10. Megreya AM, Memon A, Havard C (2012) The headscarf effect: Direct evidence from eyewitness identification paradigm. Applied Cognitive Psychology 26 308-315.

11. Deffenbacher KA, Bornstein BH, Penrod SD, McGorty K (2004) A meta-analytic review of the effects of high stress on eyewitness memory. Law \& Human

*Corresponding author: Megreya A, Department of Psychological Sciences, College of Education, Qatar University, Qatar, E-mail: amegreya@qu.edu.qa

Received: January 01 , 2016; Accepted: January 01 , 2016; Published: January 08,2016

Citation: Megreya AM (2016) Individual Differences in Face Identification: A Causal Relationship with Anxiety? . Clin Exp Psychol 2: e103. doi:10.4172/2471 2701.1000e103

Copyright: @ 2016 Megreya AM. This is an open-access article distributed under the terms of the Creative Commons Attribution License, which permits unrestricted use, distribution, and reproduction in any medium, provided the original author and source are credited. 
Citation: Megreya AM (2016) Individual Differences in Face Identification: ACausal Relationship with Anxiety? . Clin Exp Psychol 2: e103. doi:10.4172/24712701.1000e103

Page 2 of 2

Behavior 28: 687-706.

12. Megreya AM, Sandford A, Burton AM (2013) Matching face images taken on the same day or months apart: The limitations of photo-ID. Applied Cognitive Psychology 27: 700-706.

13. Davis JM, McKone E, Dennett H, O'Connor KB, O'Kearney R, et al. (2011) Individual differences in the ability to recognise facial identity are associated with social anxiety. PLoS ONE 6: e28800.

14. O'Donnell C, Bruce V (2001) Familiarisation with faces selectively enhances sensitivity to changes made to the eyes. Perception 30: 755-764.

15. Mueller JH, Bailis KL, Goldstein AG (1979) Depth of processing and anxiety in facial recognition. British Journal of Psychology 70: 511-515.

16. Attwood AS, Penton-Voak IS, Burton AM, Munafò MR (2013) Acute Anxiety Impairs Accuracy in Identifying Photographed Faces. Psychological Science 8: 1591-1594.
17. Megreya AM, Bindemann M (2012) Identification accuracy for single- and double-perpetrator crimes: Does accomplice gender matter? British Journal of Psychology 103: 439-453.

18. Megreya AM, Memon A, Havard C (2012) The headscarf effect: Direct evidence from eyewitness identification paradigm. Applied Cognitive Psychology 26: 308-315.

19. Deffenbacher KA, Bornstein BH, Penrod SD, McGorty K (2004) A metaanalytic review of the effects of high stress on eyewitness memory. Law \& Human Behavior 28: 687-706.

20. Davis JM, McKone E, Dennett H, O'Connor KB, O'Kearney R, et al. (2011) Individual differences in the ability to recognise facial identity are associated with social anxiety. PLoS ONE 6: e28800.

21. O'Donnell C, Bruce V (2001) Familiarisation with faces selectively enhances sensitivity to changes made to the eyes. Perception 30: 755-764. 